\title{
Dynein and dynactin deficiencies affect the formation and function of the Spitzenkörper and distort hyphal morphogenesis of Neurospora crassa
}

\author{
Meritxell Riquelme, ${ }^{1}$ Gerhard Gierz ${ }^{2}$ and Salomon Bartnicki-García ${ }^{1}$
} Author for correspondence: Salomon Bartnicki-García. Tel: +1 9097874135 . Fax: +1 9097874294.
e-mail: bart@ucrac1.ucr.edu

Department of Plant

Pathology ${ }^{1}$ and

Department of

Mathematics2, University

of California, Riverside,

CA 92521-0122, USA
The impact of mutations affecting microtubule-associated motor proteins on the morphology and cytology of hyphae of Neurospora crassa was studied. Two ropy mutants, ro-1 and ro-3, deficient in dynein and dynactin, respectively, were examined by video-enhanced phase-contrast microscopy and image analysis. In contrast to the regular, hyphoid morphology of wildtype hyphae, the hyphae of the ropy mutants exhibited a great variety of distorted, non-hyphoid morphologies. The ropy hyphae were slow-growing and manifested frequent loss of growth directionality. Cytoplasmic appearance, including organelle distribution and movement, were ostensibly different in the ropy hyphae. The Spitzenköper (Spk) of wild-type hyphae was readily seen by phase-contrast optics; the Spk of both ro-1 and ro-3 was less prominent and sometimes undetectable. Only the fast-growing ropy hyphae displayed a Spk, and it was smaller and less phase-dark than the wild-type Spk. Growth rate in both wild-type and ropy mutants was directly correlated with the size of the Spk. Spk efficiency, measured in terms of cell area generated per Spk travelled distance, was lower in ropy mutants. Another salient difference between ropy mutants and wild-type hyphae was in Spk trajectory. Whereas the Spk of wild-type hyphae maintained a trajectory close to the cell growth axis, the Spk of ropy hyphae moved much more erratically. Sustained departures in the trajectory of the ropy Spk produced corresponding distortions in hyphal morphology. A causal correlation between Spk trajectory and cell shape was tested with the Fungus Simulator program. The characteristic morphologies of wild-type or ropy hyphae were reproduced by the Fungus Simulator, whose vesicle supply centre (VSC) was programmed to follow the corresponding Spk trajectories. This is evidence that the Spk controls hyphal morphology by operating as a VSC. These findings on dynein or dynactin deficiency support the notion that the microtubular cytoskeleton plays a major role in the formation and positioning of the Spk, with dramatic consequences on hyphal growth and morphogenesis.

Keywords : ropy mutants, Spitzenkörper, Neurospora crassa

\section{INTRODUCTION}

This study is part of a project to elucidate the role of the Spitzenkörper (Spk) in hyphal growth and morphogenesis of higher fungi. In the present work, we used video-enhanced phase-contrast microscopy and image

Abbreviations: Spk, Spitzenkörper; VSC, vesicle supply centre. analysis to examine the behaviour of morphological mutants of Neurospora crassa affected in genes encoding microtubule-associated motor proteins.

The Spk is a dynamic body whose structure varies widely among fungi; it usually consists of an outer vesicle cloud and an inner core (López-Franco \& Bracker, 1996). In addition to vesicles (Girbardt, 1969; Grove \& Bracker, 1970), other components have 
been detected in the Spk, including amorphous or granular material of undefined nature in the core region as well as components of the cytoskeleton and ribosomes (McClure et al., 1968; Turian, 1978; Bourett \& Howard, 1991; Roberson \& Vargas, 1994; López-Franco \& Bracker, 1996). The morphology of the fungal cells is determined by the way the cell wall is assembled (Bartnicki-García, 1968, 1973). In hyphae, cell wall growth occurs mainly at the tip by polarized secretion of enzymes and cell wall precursors (Bartnicki-García \& Lippman, 1969; Trinci, 1978; Harold, 1990). There is a sizeable body of evidence that the Spk plays a central role in apical growth and morphogenesis (Girbardt, 1957; López-Franco \& Bracker, 1996; Reynaga-Peña \& Bartnicki-García, 1997). According to the vesicle supply centre (VSC) model for fungal morphogenesis (Bartnicki-García et al., 1989), the Spk functions as a vesicle distribution centre. Vesicles generated in distal parts of the hypha congregate in the Spk and from there migrate to the cell surface. The linear displacement of the Spk creates a sharp gradient of exocytosis responsible for hyphal morphogenesis.

There is mounting evidence that the position of the Spk governs the growth direction of a hypha (Girbardt, 1957; Bracker et al., 1997; Riquelme et al., 1998). Our previous work with microtubule inhibitors implicated the microtubular cytoskeleton in the positioning and movement of the Spk in hyphae of N. crassa (Riquelme et al., 1998), but its exact role in apical growth has yet to be elucidated. It has long been proposed that cytoplasmic microtubules participate in the transport of secretory vesicles to the hyphal apex (Howard \& Aist, 1977, 1980; Howard, 1981; Gooday, 1983; Gow, 1989; Hasek \& Bartnicki-García, 1994; McKerracher \& Heath, 1987; Heath, 1994). The discovery of microtubule-associated motor proteins (Hirokawa, 1982; Paschal et al., 1987) has helped us understand how organelles move inside the cell. Cytoplasmic dyneins and members of the kinesin superfamily are the main motor enzymes involved in vesicle translocation along microtubules (Haimo \& Thaler, 1994; Hirokawa, 1998). There is now growing evidence that both kinesins and cytoplasmic dyneins are involved in the traffic of secretory vesicles in fungal hyphae (Seiler et al., 1997; Wu et al., 1998; Inoue et al., 1998). Cytoplasmic dyneins are multisubunit enzymes involved in transport of membranous organelles towards the minus end of microtubules (Paschal et al., 1987; Schroer \& Sheetz, 1991; Hirokawa, 1998). Kinesins are motor proteins involved in membrane transport towards the plus end of the microtubules (Vale et al., 1985; Hirokawa, 1982; Steinberg \& Schliwa, 1996).

To examine the relationship among the Spk, the microtubular cytoskeleton and hyphal morphogenesis, we chose two ropy mutants of N. crassa, ro-1 and ro-3. Both belong to the group of true colonial morphological mutants (Garnjobst \& Tatum, 1967; Vierula, 1996) and have been characterized at the molecular level. Mutant ro-1 is deficient in one of the heavy chains of cytoplasmic dynein (Plamann et al., 1994). Mutant ro-3 is deficient in $\mathrm{p} 150^{\text {Glued }}$, the largest subunit of the dynactin (dynein activator) complex (Plamann et al., 1994; Tinsley et al., 1996). Dynactin is a multisubunit complex required for cytoplasmic dynein to efficiently transport vesicles along microtubules in vitro (Gill et al., 1991; Schroer \& Sheetz, 1991).

Most studies on ropy mutants have primarily focused on the aberrant distribution of nuclei in ro-1 and ro-3 hyphae and on the molecular characterization of those loci (Plamann et al., 1994; Bruno et al., 1996; Tinsley et al., 1996). Since hyphal and, ultimately, colony morphology are largely established at the hyphal apex, we have focused this study on the impact of the ropy mutations on apical events, where most of the growth process is concentrated.

\section{METHODS}

Strains and media. N. crassa wild-type (FGSC 988) and ropy strains (FGSC 4351 and 43 for ro- 1 and ro-3 respectively) were obtained from the Fungal Genetics Stock Center. The strains were grown and maintained in $8.5 \mathrm{~cm}$ plastic Petri dishes (sold as $100 \times 15 \mathrm{~mm}$, Fischer Scientific) containing $20 \mathrm{ml}$ Vogel's Complete Medium (VCM) agar (Vogel, 1956) with 1.5\% $(\mathrm{w} / \mathrm{v})$ sucrose as the carbon source. Cultures were grown at $21^{\circ} \mathrm{C}$.

Video microscopy. Growing hyphae were observed with an Olympus Vanox-S microscope. For low-magnification images, the fungus was grown in $8.5 \mathrm{~cm}$ plastic Petri dishes on a thin layer $(10 \mathrm{ml})$ of VCM agar at $21^{\circ} \mathrm{C}$ and observed with brightfield optics $(10 \times$ objective and $10 \times$ WF eyepiece). For highresolution work, the fungus was grown on a modified slide culture chamber (López-Franco, 1992; Riquelme et al., 1998) and observed with a phase-contrast $100 \times$ oil-immersion objective (n.a. 1.25) and a $25 \times$ WF eyepiece (American Optical).

Video images were produced with a Hamamatsu C2400-07 high-resolution camera (Hamamatsu Photonic Systems), enhanced with an Argus-10 image processor (a real-time digital contrast and low light enhancement system), and displayed on a black and white, 12-inch, high-resolution monitor (Sony; model PVM-122). Sequences were videotaped in real time with an S-VHS recorder (JVC model BR-S822U).

Growth rate and cell parameters measurements. Videotaped sequences were played on a variable-tracking player (JVC model BR-S525U) and observed on a Sony Trinitron model monitor. Individual images were captured from the videotaped sequences in 8-bit greyscale with an Imascan/Chroma frame grabber (Imagraph). With Image Pro Plus Software for Windows (Media Cybernetics), we traced the cell profiles of the images captured by video microscopy. $x y$ coordinate values were automatically collected into a text file with a Windows application program interfaced with the Argus-10 analyser (Bartnicki et al., 1994). The text files were then imported into Microsoft Excel spreadsheets and analysed.

Growth rate was measured in terms of cell area increase. Typically, measurements were made during growth periods of 1-4 min depending on growth rate and availability of welldefined profiles. For convenience and accuracy, only images within the same field of view were compared. The area delimited by the cell profiles was computed by using Green's formula (Marsden et al., 1993). Spk diameter was measured 

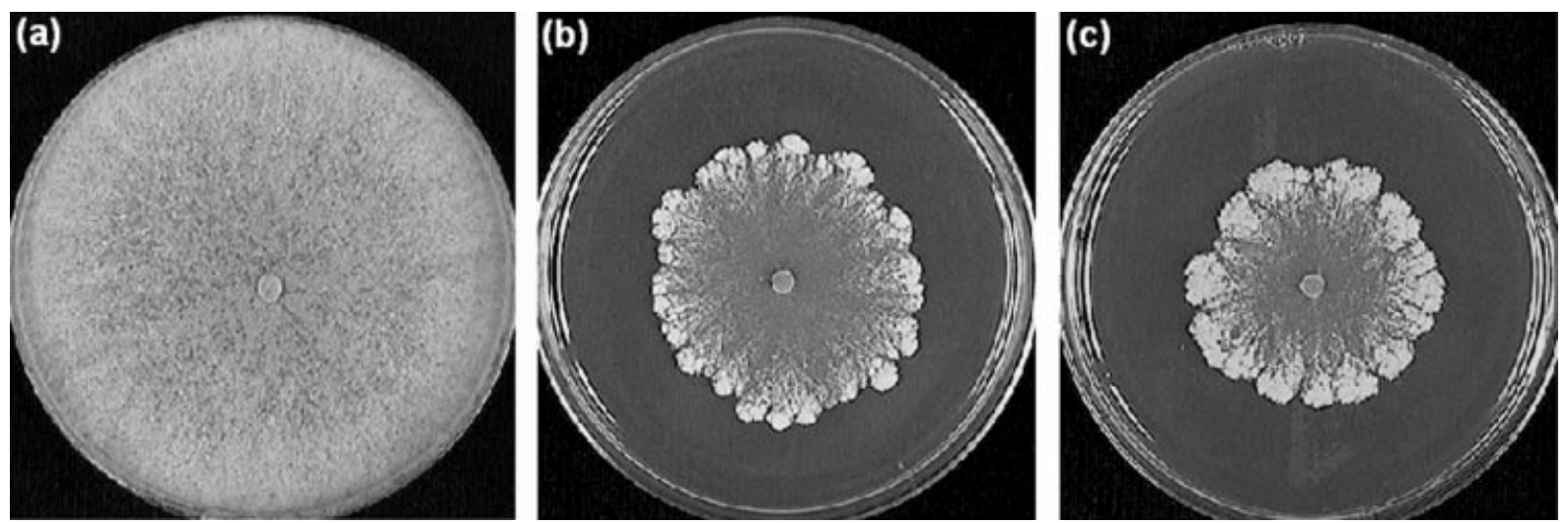

Fig. 1. Colonies of $N$. crassa wild-type (a), ro-1 (b) and ro-3 (c) grown on VCM (20 ml) for $24 \mathrm{~h}$ at $28^{\circ} \mathrm{C}$.

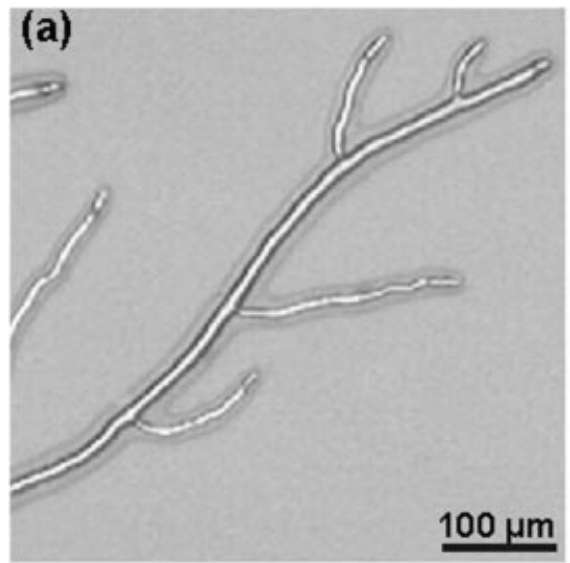

(b)
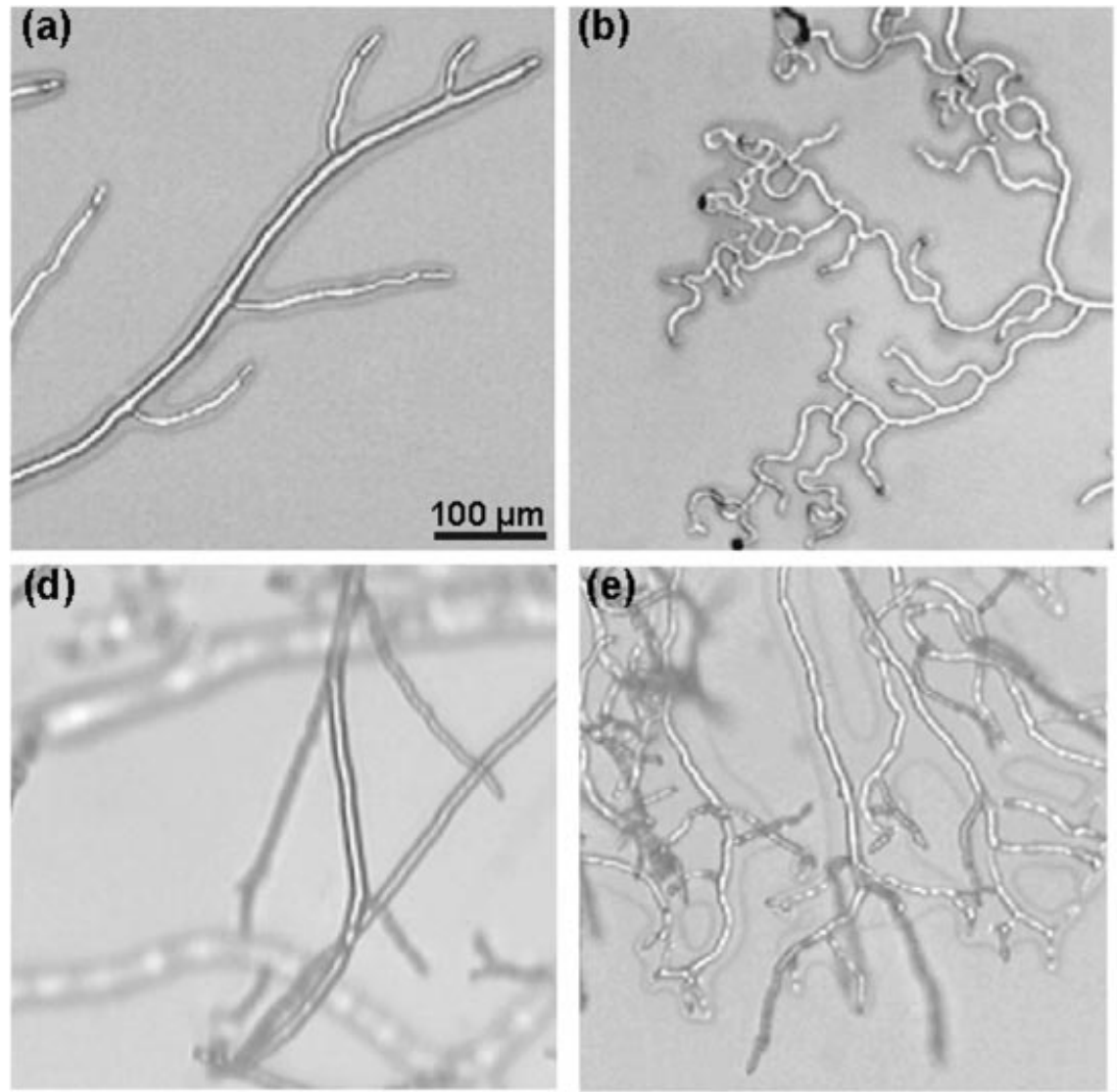

(c)
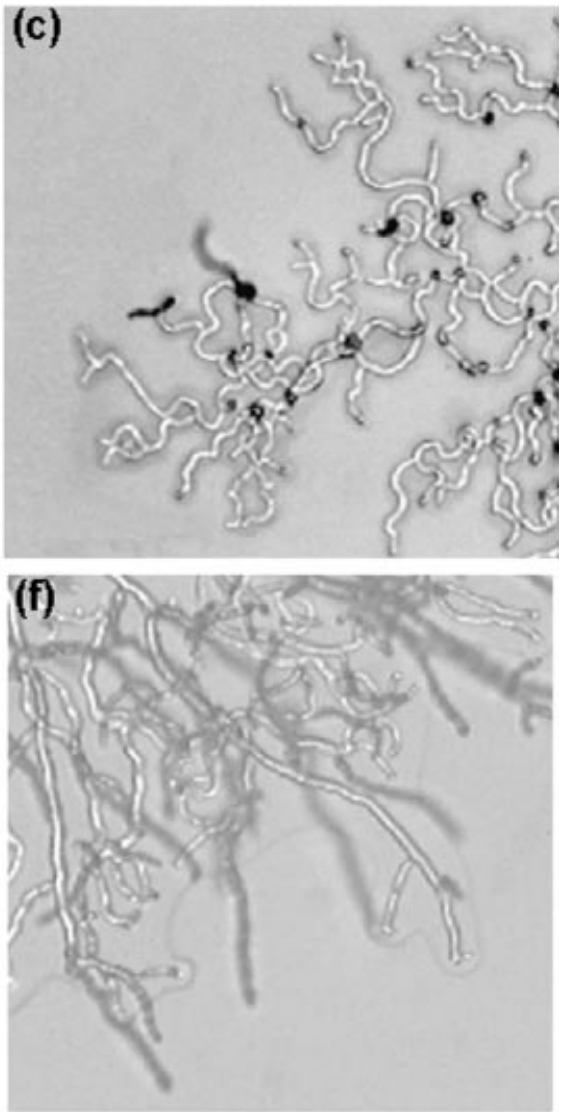

Fig. 2. Bright-field micrographs of hyphae of $N$. crassa wild-type (a, d), ro-1 (b, e) and ro-3 (c, f). Top row after $14 \mathrm{~h}$ growth; bottom row after $24 \mathrm{~h}$ growth.

directly on the monitor screen with the line command of the measure option in the Argus-10 menu.

Spk trajectory analysis. The centre of the Spk was mapped at 2 s intervals from the videotaped sequences. To quantitate the erratic behaviour of the advancing Spk, we calculated a steadiness index $(S)$, namely the ratio of the minimal distance between the initial and final position of the Spk in a given videotaped sequence over the total distance travelled by the
Spk. A steadiness index of 1 would correspond to a perfectly straight path. The smaller the steadiness index the less steady the trajectory of the Spk.

Computer simulation. From videotaped sequences of hyphae of wild-type and ropy mutants, we mapped the Spk position (every $2 \mathrm{~s}$ ) and traced the cell profiles at various intervals. These data were fed to the Fungus Simulator [a Windows program for fungal morphogenesis (Bartnicki et al., 1994) 
available through the Internet (http://boyce3427.ucr.edu)]. The Fungus Simulator generates hyphal shapes by a process that mimics a vesicle-based mechanism for cell wall growth.

\section{RESULTS}

\section{Colony and hyphal morphology}

On agar plates, the two ropy mutants formed colonies that were drastically different from the wild-type. Radial growth was restricted and the aerial mycelium showed the characteristic ropy phenotype (Fig. 1).

Under the microscope, the ropy mutants showed remarkable differences in hyphal morphology compared to the wild-type (Fig. 2). In a young colony (8-14 h), the morphology of ropy hyphae was highly irregular, with extensive curling - an indication of a major loss in growth directionality (Fig. 2 b, c). In contrast, the hyphae of the wild-type were more regular, and despite some minor meandering they exhibited long stretches of mostly straight growth (Fig. 2a). In older colonies ( $>14 \mathrm{~h}$ ), the morphology of hyphae growing on the agar surface remained the same but the ropy mutants produced large cottony tufts of aerial hyphae at the periphery of the colony. This aerial mycelium consisted of some very long and straight primary hyphae (Fig. $2 \mathrm{e}, \mathrm{f})$ with numerous short curly branches. In the wildtype, mycelium spread faster on the agar surface, producing a flatter and more uniform mat of aerial hyphae. Both surface and aerial hyphae grew rather straight (Fig. 2d).

At high magnification, most wild-type hyphae (Fig. $3 \mathrm{a}, \mathrm{b})$ showed a regular apical profile that approximated the ideal shape defined by the hyphoid equation $y=x$ $\cot (x \mathrm{~V} / \mathrm{N})$. There were also some slow-growing wildtype hyphae that deviated appreciably from this hyphoid morphology (Fig. 3c). In contrast, the hyphae of the ropy mutants exhibited a great variety of distorted, nonhyphoid morphologies: some apices were blunt, others more pointed; often, the hyphal profile was highly irregular with conspicuous swellings (Fig. 3d-i).

\section{Cytoplasmic appearance}

As visualized by phase-contrast microscopy (Fig. 3), there were major differences in cytoplasmic appearance between hyphae of the ropy mutants and wild-type. In the apex of wild-type hyphae, the dominant structure was a large phase-dark Spk (Fig. 3a, b) that corresponds to the organizational pattern 8 described by López-
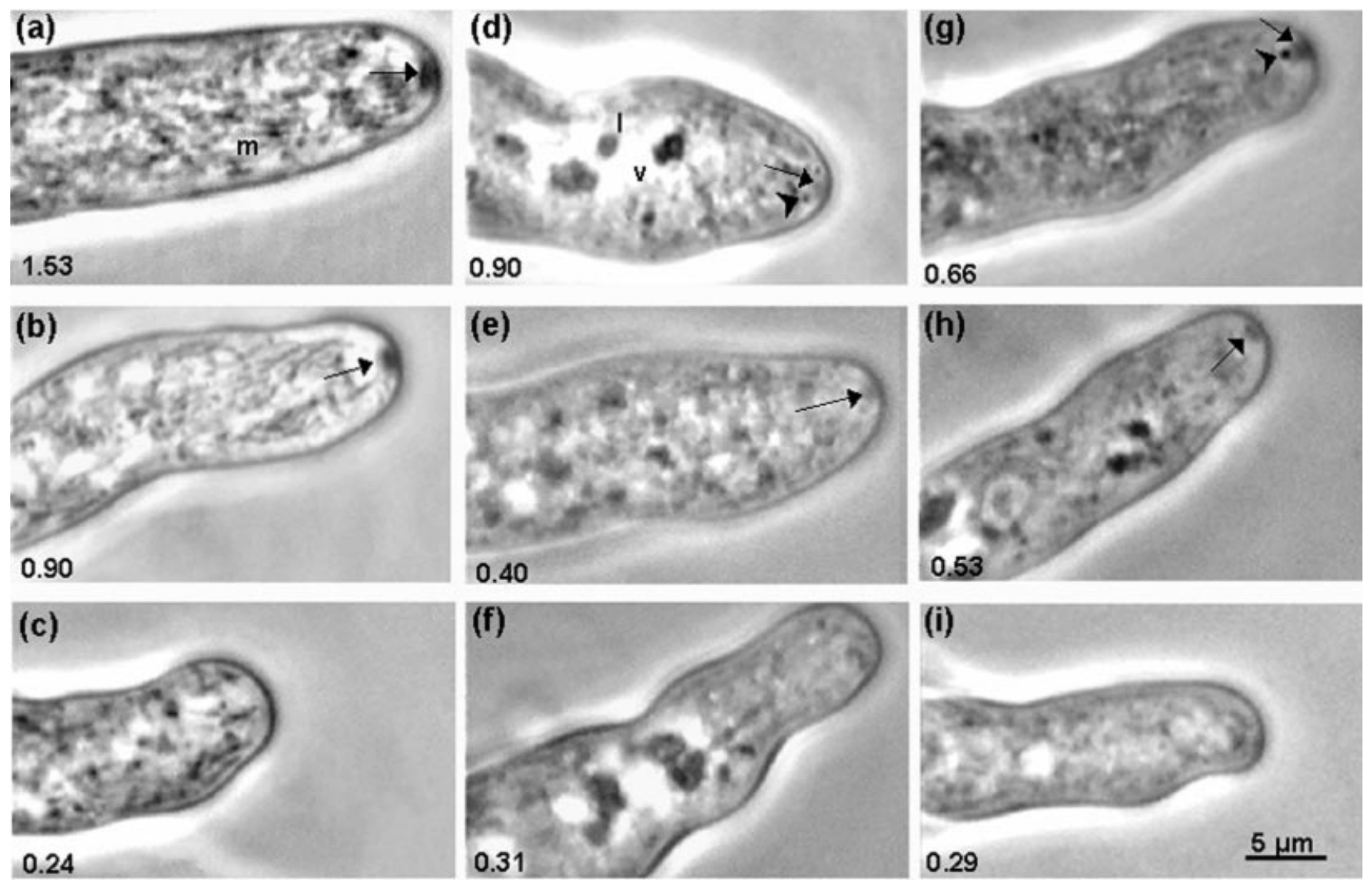

Fig. 3. Variation in hyphal tip morphology in wild-type and ropy mutants of $N$. crassa. Phase-contrast micrographs of wild-type (a-c), ro-1 (d-f) and ro-3 (g-i) hyphae. Numbers indicate growth rate in $\mu^{2} \mathrm{~s}^{-1}$. Arrows, Spk; m, mitochondria; v, vacuoles; I, lipid bodies. Arrowheads (in d and g) point at a phase-dark globular body. 
Table 1. Cell parameters of $N$. crassa wild-type, ro-1 and ro-3

Data are mean values \pm SD.

\begin{tabular}{|c|c|c|c|c|}
\hline Strain* & $\begin{array}{l}\text { No. of } \\
\text { hyphae } \\
\text { analysed }\end{array}$ & $\begin{array}{c}\text { Growth rate } \\
\qquad\left(\mu \mathrm{m}^{2} \mathrm{~s}^{-1}\right)\end{array}$ & $\begin{array}{c}\text { Hyphal } \\
\text { diameter } \\
(\mu \mathrm{m}) \dagger\end{array}$ & $\begin{array}{c}\text { Spk } \\
\text { diameter } \\
(\mu \mathrm{m}) \neq\end{array}$ \\
\hline \multirow[t]{5}{*}{ Wild-type } & 4 & $3 \cdot 47 \pm 0 \cdot 63$ & $10 \cdot 67 \pm 1 \cdot 03$ & $1 \cdot 85 \pm 0 \cdot 14$ \\
\hline & 4 & $1 \cdot 42 \pm 0 \cdot 20$ & $9 \cdot 82 \pm 0 \cdot 60$ & $1 \cdot 35 \pm 0 \cdot 15$ \\
\hline & 4 & $0 \cdot 84 \pm 0 \cdot 07$ & $8 \cdot 28 \pm 0 \cdot 88$ & $1 \cdot 32 \pm 0 \cdot 30$ \\
\hline & 4 & $0 \cdot 61 \pm 0 \cdot 16$ & $7 \cdot 36 \pm 1.97$ & $1 \cdot 05 \pm 0.32$ \\
\hline & 3 & $0 \cdot 28 \pm 0 \cdot 04$ & $8.92 \pm 1.98$ & $\mathbb{S}$ \\
\hline \multirow[t]{5}{*}{ ro-1 } & 5 & $1 \cdot 38 \pm 0 \cdot 48$ & $8 \cdot 96 \pm 1 \cdot 54$ & $1 \cdot 02 \pm 0 \cdot 31$ \\
\hline & 5 & $0.77 \pm 0.06$ & $7 \cdot 19 \pm 0 \cdot 48$ & $0 \cdot 82 \pm 0 \cdot 23$ \\
\hline & 5 & $0 \cdot 50 \pm 0 \cdot 07$ & $8 \cdot 29 \pm 2 \cdot 36$ & $0 \cdot 93 \pm 0 \cdot 28$ \\
\hline & 4 & $0 \cdot 24 \pm 0 \cdot 10$ & $6 \cdot 60 \pm 0 \cdot 82$ & $0 \cdot 67 \pm 0.36$ \\
\hline & 3 & $0 \cdot 09 \pm 0.02$ & $6 \cdot 97 \pm 2 \cdot 70$ & $\mathbb{S}$ \\
\hline \multirow[t]{5}{*}{ ro-3 } & 5 & $0 \cdot 83 \pm 0 \cdot 22$ & $8 \cdot 02 \pm 0 \cdot 89$ & $0 \cdot 81 \pm 0 \cdot 12$ \\
\hline & 4 & $0.57 \pm 0.03$ & $7 \cdot 85 \pm 0 \cdot 46$ & $0 \cdot 96 \pm 0 \cdot 12$ \\
\hline & 4 & $0.41 \pm 0.08$ & $7 \cdot 13 \pm 1 \cdot 24$ & $0 \cdot 84 \pm 0 \cdot 05$ \\
\hline & 4 & $0 \cdot 29 \pm 0 \cdot 04$ & $7 \cdot 05 \pm 1 \cdot 44$ & $0 \cdot 72 \pm 0.04$ \\
\hline & 2 & $0 \cdot 15 \pm 0 \cdot 03$ & $5 \cdot 69 \pm 0 \cdot 12$ & $\mathbb{S}$ \\
\hline
\end{tabular}

*For each strain, the values for individual hyphae (Fig. 4) were organized in subgroups in descending order of growth rate.

† Hyphal diameter was calculated by dividing the hyphal area by the length of the hypha.

$\ddagger$ During each growth sequence, Spk diameter was measured when it was most clearly defined.

$\$$ No Spk was visible.

Franco \& Bracker (1996). In the ropy mutants, the Spk was smaller and less phase-dark (Fig. 3d, e, g, h). In the subapex, there were also marked differences between wild-type and ropy. In general, organelle distribution was more uniform in the wild-type. A remarkable difference was in the appearance of mitochondria. In the wild-type strain, these long, phase-dark organelles were the dominant structure of the cytoplasm. In the ropy mutants, the mitochondria were round and less conspicuous, giving the cytoplasm a more finely granular appearance, particularly in slower-growing hyphae.

Another common difference was in vacuolation. The cytoplasm of both ropy mutants was more vacuolated than in wild-type. In ropy hyphae, large, phase-light and phase-dark structures of irregular shape (probably vacuoles and lipid bodies) accumulated at a shorter distance (as close as $10-15 \mu \mathrm{m}$ ) from the tip. Occasionally, one or more small phase-dark globular bodies were seen immediately behind the Spk (Fig. $3 \mathrm{~d}, \mathrm{~g}$; arrowheads). These bodies moved continuously within the area surrounding the Spk but appeared to be physically attached to the Spk by thin filaments and remained behind the Spk for the entire observation period. These granules were usually more conspicuous in the ropy mutants but were some times seen in tips of wild-type hyphae. Similar structures have been seen in other fungi (López-Franco \& Bracker, 1996) but their function remains unknown.

There was a pronounced difference in overall movement of cytoplasmic structures between wild-type and ropy mutants. To judge differences in organelle movement, the videotaped growth sequences were examined in real time and at three times the original speed. The cytoplasm in the hyphae of ropy mutants was ostensibly less dynamic than that in wild-type hyphae. In the latter, cytoplasmic organelles were in constant motion over the entire length of the subapical area observed (at least 20$25 \mu \mathrm{m}$ from the apex). In ropy hyphae, motion was less active and was usually restricted to the proximal subapex $(5-15 \mu \mathrm{m}$ from the apex); beyond that, the cytoplasm became static and extensively vacuolated (Fig. 3; see also video in http://boyce3427.ucr.edu/ cytoskel.htm). In both ropy mutants, the movement of cytoplasmic structures appeared less organized than that in wild-type hyphae. In growing wild-type hyphae, the mitochondria moved continuously in a back and forth fashion roughly parallel to the longitudinal axis of growth. In the ropy mutants, mitochondria did not move preferentially along the longitudinal axis but in a more erratic manner.

Table 2. Cell parameters of $N$. crassa wild-type, ro-1 and ro-3

Values for each strain are the mean of all cells analysed in Table 1. Means within each column followed by the same letter are not significantly different $(P=0.05)$ according to Student's $t$-test analysis.

\begin{tabular}{|c|c|c|c|c|c|c|c|}
\hline \multirow[t]{2}{*}{ Strain } & \multirow{2}{*}{$\begin{array}{l}\text { No. of } \\
\text { hyphae } \\
\text { analysed }\end{array}$} & \multirow{2}{*}{$\begin{array}{l}\text { Growth } \\
\text { rate } \\
\left(\mu \mathrm{m}^{2} \mathrm{~s}^{-1}\right)\end{array}$} & \multirow{2}{*}{$\begin{array}{l}\text { Hyphal } \\
\text { diameter } \\
\quad(\mu \mathrm{m})\end{array}$} & \multirow{2}{*}{$\begin{array}{l}\text { Spk diameter } \\
\qquad(\mu \mathrm{m})\end{array}$} & \multicolumn{3}{|c|}{ Ratios } \\
\hline & & & & & $\begin{array}{c}\text { Growth } \\
\text { rate/hyphal } \\
\text { diameter }\end{array}$ & $\begin{array}{l}\text { Growth } \\
\text { rate/Spk } \\
\text { diameter }\end{array}$ & $\begin{array}{c}\text { Hyphal } \\
\text { diameter/Spk } \\
\text { diameter }\end{array}$ \\
\hline Wild-type & 19 & $1 \cdot 40^{a}$ & $9 \cdot 94^{a}$ & $1 \cdot 37^{a}$ & $0 \cdot 13$ & $1 \cdot 01$ & $7 \cdot 66$ \\
\hline ro-1 & 24 & $0 \cdot 63^{b}$ & $7 \cdot 21^{b}$ & $0 \cdot 87^{b}$ & 0.09 & $0 \cdot 87$ & $9 \cdot 02$ \\
\hline ro-3 & 20 & $0 \cdot 50^{b}$ & $6 \cdot 54^{b}$ & $0 \cdot 83^{b}$ & $0 \cdot 07$ & $0 \cdot 65$ & $8 \cdot 17$ \\
\hline
\end{tabular}



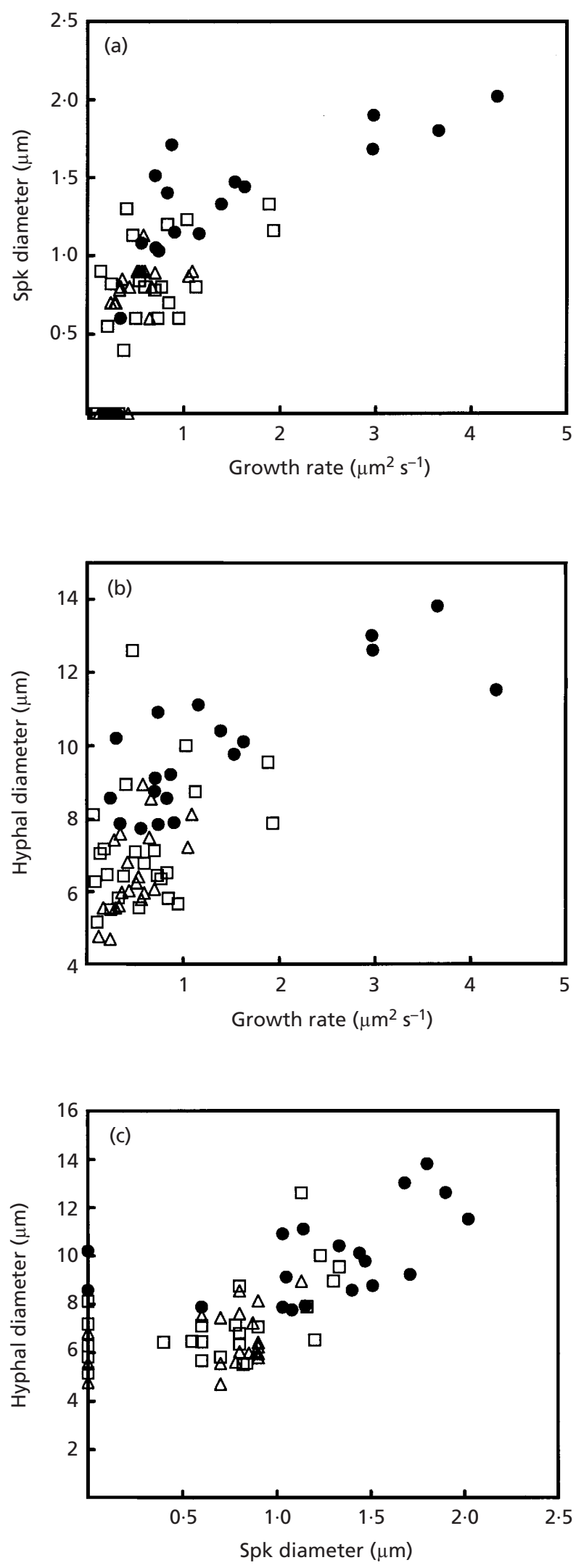

Fig. 4. Correlation between growth rate, hyphal diameter and Spk diameter in hyphae of $N$. crassa wild-type $(\bullet)$, ro-1 $(\square)$ and ro-3 $(\triangle)$.

\section{Growth rate and Spk size}

Hyphal elongation is traditionally used as a measurement of the growth rate. This method is accurate provided the hypha maintains a relatively constant shape during elongation. For the ropy mutants, with their distorted hyphal morphology, this method was clearly not valid. Instead, we measured growth rate as increase in cell area per unit of time (Table 2) and calculated that the mean growth rate of hyphae of ro-1 and ro-3 was 45 and $34 \%$, respectively, of that in wildtype hyphae. There was no significant difference in growth rate between the two ropy mutants (Table 2).

Growth rate was correlated with the presence and size of the Spk in hyphae of both wild-type and the ropy mutants (Table 1; Fig. 4a). In general, faster-growing hyphae exhibited a larger Spk; this was true for both the wild-type and the mutants. A large well-defined Spk was the rule in wild-type hyphae (Figs 3a, b); only a few hyphae with growth rates lower than $0.35 \mu \mathrm{m}^{2} \mathrm{~s}^{-1}$ (i.e. $<10 \%$ the rate of the fastest-growing hyphae) failed to exhibit a Spk (Fig. 3c). In the two ropy mutants, even the fastest-growing hyphae tended to show a Spk that was less phase-dark and smaller than the wild-type (Fig. 3d, $\mathrm{e}, \mathrm{g}, \mathrm{h})$. Slow-growing hyphae showed only a very small Spk and none was observed in the slowest hyphae. On average, the Spk diameter of ro- 1 and ro-3 was $62 \%$ and $60 \%$, respectively, of that in wild-type (Table 2). Expressed as cross-sectional area, the relative size of the Spk of ro- 1 and ro-3 becomes $46 \%$ and $41 \%$, respectively, of the wild-type Spk, values that approximate the comparative growth rates calculated above.

Hyphal growth rate and Spk size were further correlated with hyphal diameter in both wild-type and ropy mutants (Tables 1 and 2, Fig. 4b, c). In ro-1 and ro-3 hyphae, hyphal diameter was difficult to assess because of the irregularities of the hyphal shape. Therefore, we estimated mean diameters by dividing hyphal area by hyphal length. In the wild-type hyphae, there was a positive correlation between growth rate and hyphal diameter. The faster-growing hyphae, which had a large Spk, had the largest diameter (Table 1, Fig. 4b). In the mutants, the same tendency could be observed (Table 1, Fig. 4b).

The appearance and size of the Spk varied greatly during growth, particularly in the ropy mutants. For instance, during the observation of a hyphal tip of $N$. crassa ro-1 growing at $0 \cdot 6-0 \cdot 7 \mu \mathrm{m}^{2} \mathrm{~s}^{-1}$, a smaller and less phasedark Spk was visible for about $220 \mathrm{~s}$ (Fig. 5), then the Spk disappeared, coinciding with a sharp decrease of the growth rate to $0 \cdot 1 \mu \mathrm{m}^{2} \mathrm{~s}^{-1}$. When the growth rate returned to almost the original values $\left(0 \cdot 5 \mu \mathrm{m}^{2} \mathrm{~s}^{-1}\right)$, the Spk became visible again.

\section{Spk trajectory}

The Spk of wild-type hyphae of N. crassa follows an intricate trajectory produced by a dominant forward motion accompanied by frequent, short, transverse 


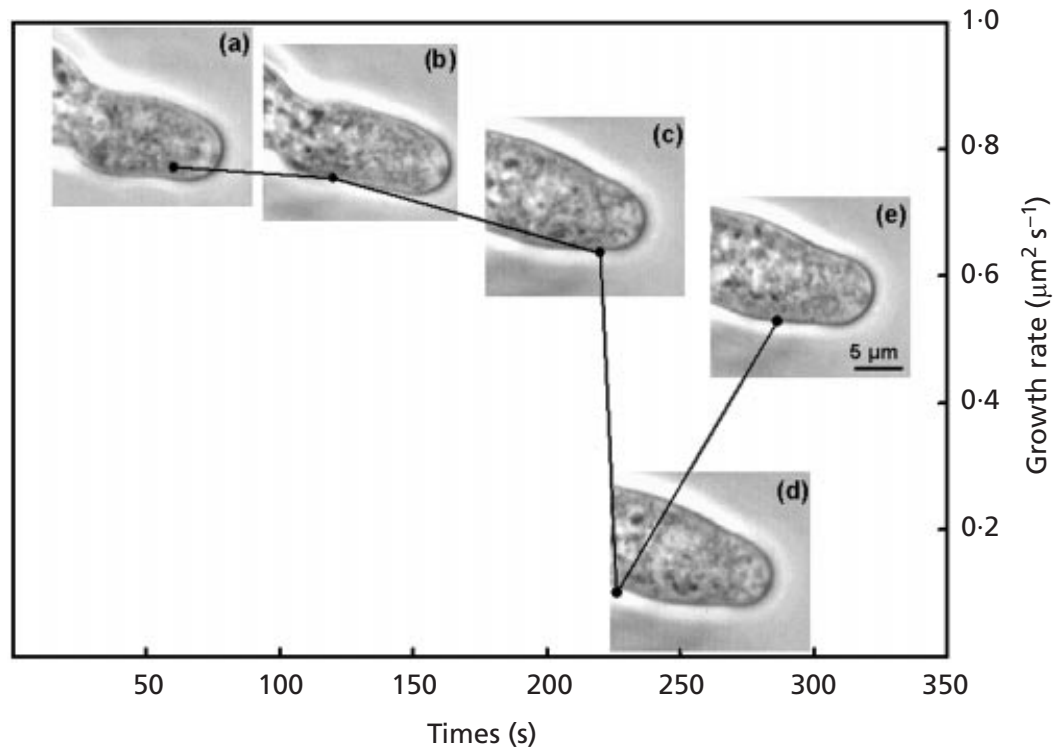

Fig. 5. Growth rate and Spk changes in a hypha of $N$. crassa ro-1. The small Spk observed from (a) to (c) is no longer visible in (d), coinciding with a drastic decrease of the growth rate. As growth rate recovers, a small Spk starts being visible again (e). (a)

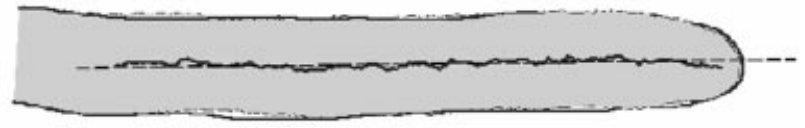

(b)

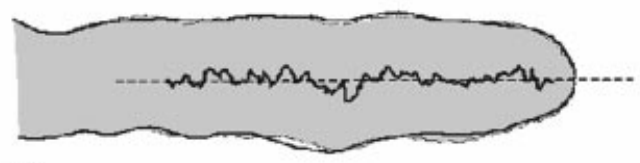

(c)

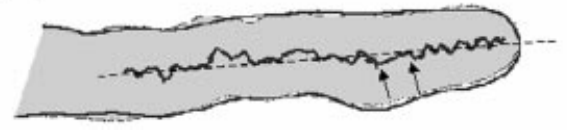

Fig. 6. Computer simulation of hyphal morphogenesis in wildtype and ropy mutants. The grey areas are the shapes generated by the Fungus Simulator programmed to follow the Spk paths of (a) wild-type hypha growing for $384 \mathrm{~s}$; (b) ro-1 hypha growing for $514 \mathrm{~s}$; (c) ro-3 hypha growing for $491 \mathrm{~s}$. For each hypha, the cell profile (dark solid line) was reconstructed by assembling profiles from three screen displacements. A correction factor was included to compensate for the slight tendency of the microscope stage to drift during the course of the observations (Bracker, 1995). The straight dashed line corresponds to the axis of growth of the cell. Arrows in (c) indicate a data gap in Spk trajectory that occurred during manipulation of the microscope stage. The gap (about $30 \mathrm{~s}$ ) was filled with a straight line for the simulation. The percentage of mismatch between simulated shape and real shape was $4.5 \%$ for wild-type, $4.2 \%$ for ro-1, and $6.05 \%$ for ro-3.

oscillations. The trajectory tends to follow the longitudinal cell axis (Fig. 6a). In the distorted hyphae of the ropy mutants, the Spk advances with an erratic trajectory that also tends to follow the longitudinal cell axis but with larger and longer-lasting departures (Fig. 6b, c). Minor oscillations in Spk trajectory cancelled each other and had no apparent impact on the cell profile. However, larger departures in the trajectory of the Spk resulted in corresponding distortions in the morphology of the hypha.

We compared the behaviour of the Spk in ropy vs wildtype hyphae by analysing trajectory and growth efficiency. To compare trajectories, we calculated the steadiness index $(S)$ (see Methods). In general, the $S$ values for wild-type hyphae were higher than those of the ropy mutants hyphae (Table 3). In the wild-type, the $S$ values were close to 1 , indicating a rather steady Spk path, whereas in the ropy mutants, the $S$ values were considerably lower, indicating a much more erratic movement of the Spk.

To compare Spk efficiency, we assessed the increase in hyphal area per total distance travelled by the Spk and also the rate of Spk advance (total distance per unit time). By both criteria, the ropy Spk was less efficient than the wild-type. In area generated per travelled distance, the ro-1 and ro-3 Spk were only $71 \%$ and $60 \%$, respectively, as efficient as the wild-type Spk (Table 3). In rate of advance, the values were $83 \%$ and $72 \%$. These two criteria combined gave overall efficiencies of $59 \%$ and $43 \%$ compared to wild-type. The latter values correspond to the overall growth rate differences between wild-type and ropy mutants (Table 3).

\section{Computer simulation}

To analyse the effect of Spk movement on hyphal morphogenesis, the traced Spk trajectories and cell profiles of wild-type and ropy mutants were fed to the Fungus Simulator program. The VSC of the simulator was programmed to follow actual Spk trajectories. Cell profiles were used to calculate the amount of vesicles to be released by the VSC at each point in the trajectory. With these two sets of input data, the simulator 
Table 3. Spk behaviour and efficiency in three representative hyphae of $N$. crassa wild-type, ro-1 and ro-3

The hyphae analysed are those in Fig. 6.

\begin{tabular}{|c|c|c|c|c|c|c|c|c|c|}
\hline \multirow[t]{2}{*}{ Strain } & \multicolumn{3}{|c|}{ Hyphal growth rate } & \multicolumn{3}{|c|}{ Spk steadiness } & \multicolumn{3}{|c|}{ Spk efficiency } \\
\hline & $\begin{array}{c}\text { Total } \\
\text { increase of } \\
\text { area }\left(\mu \mathrm{m}^{2}\right)\end{array}$ & $\begin{array}{c}\text { Time } \\
(\mathrm{s})\end{array}$ & $\begin{array}{l}\text { Growth } \\
\text { rate } \\
\left(\mu \mathrm{m}^{2} \mathrm{~s}^{-1}\right)\end{array}$ & $\begin{array}{l}\text { Net distance } \\
\text { between Spk } \\
\text { initial and final } \\
\text { position }(\mu \mathrm{m})\end{array}$ & $\begin{array}{c}\text { Total Spk } \\
\text { travelled } \\
\text { distance }(\mu \mathrm{m})\end{array}$ & $\begin{array}{c}\text { Steadiness } \\
\text { index }(S)\end{array}$ & $\begin{array}{c}\text { Area/total } \\
\text { Spk travelled } \\
\text { distance }(\mu \mathrm{m})\end{array}$ & $\begin{array}{c}\text { Total Spk } \\
\text { travelled } \\
\text { distance/time } \\
\left(\mu \mathrm{m} \mathrm{s}^{-1}\right)\end{array}$ & $\begin{array}{l}\text { Overall } \\
\text { efficiency }\end{array}$ \\
\hline Wild-type & 536 & 384 & $1 \cdot 40$ & $53 \cdot 9$ & $60 \cdot 4$ & 0.89 & 8.87 & $0 \cdot 16$ & $1 \cdot 40$ \\
\hline ro-1 & 472 & 514 & $0 \cdot 92$ & $35 \cdot 0$ & 67 & 0.52 & $7 \cdot 04$ & $0 \cdot 13$ & 0.92 \\
\hline ro-3 & 315 & 491 & 0.64 & 33.7 & $54 \cdot 7$ & 0.62 & $5 \cdot 75$ & $0 \cdot 11$ & 0.64 \\
\hline
\end{tabular}

*Overall efficiency is the product of (Area/total Spk travelled distance) times (Total Spk travelled distance/time).

duplicated the morphologies of wild-type hyphae and the highly distorted shapes of ropy mutants hyphae (Fig. 6).

\section{DISCUSSION}

High-resolution video microscopy has allowed us to analyse quantitatively key cellular details of the phenotype of two ropy mutants of N. crassa (ro-1 and ro-3). We focused this study on the effects of these mutations (dynein and dynactin, respectively), on the behaviour of the Spk and its consequences on hyphal growth and morphogenesis.

Impairment of dynein or dynactin caused major effects at different levels: reduced growth rate, distorted morphology, aberrant cytoplasmic organization, disrupted organelle motility, and a smaller Spk with erratic trajectory. The effects of these mutations on nuclear distribution and mitosis have been previously documented for this and other fungi (Xiang et al., 1994, 1995 ; Plamann et al., 1994; Tinsley et al., 1996; Bruno et al., 1996; Inoue et al., 1998). Apparently, the reduction in the overall movement of intracellular components along the microtubular cytoskeleton, caused by dynein/ dynactin deficiencies, had a general effect on all cytoplasmic activities and hence led to an overall reduction in growth rate. But diminished growth rate need not result in distorted morphology; it should result in slower-growing hyphae with similar morphology. We believe the morphogenetic effects caused by these mutations can be directly ascribed to their impact on Spk formation and behaviour. Invariably, the Spk of ropy hyphae was smaller and lacked the stability of the wild-type Spk. The fact that the ropy mutants grew poorly and produced deformed hyphae on Petri dish cultures in the dark eliminates the possibility that the Spk deficiencies we observed were caused by stresses imposed on the mutants during microscopy.

\section{Spk size}

Presumably, the smaller size of the Spk of ropy hyphae is a consequence of a diminished supply of vesicles to the apical region caused by the dynein/dynactin deficiency in the mutants. This conclusion differs from that made by Seiler et al. (1999), who described the ro-1 mutant as having a 'prominent' Spk. However, they apparently did not take into account that the Spk of ro-1 is considerably smaller than that of the wild-type, and at times may not even be visible, as shown here in Fig. 4 (see also http://boyce3427.ucr.edu/cytoskel.htm for video). Consequently, their conclusion that 'apical transport was intact' in this ropy mutant runs contrary to our evidence, which clearly shows that the processes responsible for Spk formation, including apical vesicle traffic, are affected by dynein deficiency. Inoue et al. (1998) also found that dynein is required for normal secretory vesicle transport to the hyphal apex of Nectria haematococca.

Since mutations in kinesin are known to affect Spk formation (Seiler et al., 1997; Wu et al., 1998), it was proposed that cytoplasmic microtubules are oriented with their plus ends towards the apex (Lehmler et al., 1997; Seiler et al., 1997, 1999). By the same reasoning, our studies showing that deficiency in cytoplasmic dynein or dynactin affects Spk formation could lead us to the opposite conclusion, namely that the microtubules are oriented with their minus ends towards the apex. Clearly, none of these observations can reveal conclusively the polarity of cytoplasmic microtubules in a hypha. The similarity of phenotypic effects caused by the impairment of opposite motor proteins suggests that both motors are necessary for the maintenance of the Spk and apical growth, but whether or not both are directly involved in the apical transport of secretory vesicles remains to be seen. Possibly, a deficiency in cytoplasmic dynein or kinesin may also impair the endocytotic processes that contribute to the recycling of membranous components from apex to subapex (Hoffmann \& Mendgen, 1998), and thus affect Spk formation.

Overall, our analyses showed positive, though not necessarily linear, correlations between Spk size, hyphal growth rate and hyphal diameter in both the wild-type and the ropy mutants of $N$. crassa. In general, fastgrowing hyphae share a tendency to have a larger hyphal diameter and a larger Spk than slow-growing hyphae. 
Similar tendencies were observed in other fungi (LópezFranco \& Bracker, 1996). Wu et al. (1998) found a similar correlation between Spk size, growth rate and hyphal diameter in a kinesin-deletion mutant of Nectria haematococca. In other tip-growing cells such as pollen tubes and root hairs, the size of what would be their Spk equivalent (so-called tip body or clear cap) has also been correlated with high growth rate (Reiss \& Herth, 1979; Sievers, 1963).

\section{Spk behaviour and morphogenesis}

The highly erratic behaviour of the Spk in ropy mutants is difficult to interpret since we do not know for sure which cellular components determine the positioning and advance of such a complex and dynamic structure as the Spk. Previously, based on comparative results with benomyl and cytochalasin, we proposed that the microtubule cytoskeleton was directly involved in maintaining the trajectory of the Spk (Riquelme et al., 1998). Our present observations with the dynein-deficient ropy mutants lend support to the notion that the microtubule cytoskeleton plays a major role in the formation and behaviour of the Spk. Similarly, Wu et al. (1998) showed that kinesin was essential for normal positioning of the Spk in hyphae of N. haematococca. All this leads us to conclude that microtubule-associated motor proteins are necessary for maintenance of a high growth rate, a rather steady Spk and a near-perfect hyphoid shape.

Regardless of the exact mechanism controlling the position of the Spk, we have reason to conclude that the erratic trajectory of the Spk is directly responsible for the distorted morphology of ropy hyphae. As we did in studying other morphogenetic processes (BartnickiGarcía et al., 1995; Reynaga-Peña \& Bartnicki-García, 1997; Riquelme et al., 1998), we used the Fungus Simulator to test the correlation between Spk trajectory and cell shape. The simulator generated forms that reproduced the distorted morphology of the ropy hyphae. This indicates that the fungal Spk, operating as a VSC, is the structure that ultimately controls the shape of fungal hyphae. In wild-type hyphae, the Spk advances in a fixed direction with only minor transverse oscillations. The spatially uniform vesicle traffic emanating from such a Spk produces a smooth regular shape that approximates the ideal 'hyphoid' shape stipulated by the hyphoid equation (Bartnicki-García et al., 1989). In the ropy hyphae, sustained departures in the trajectory of the Spk result in corresponding distortions in the morphology of the hypha.

Barring pleiotropic effects caused by the ropy mutations, the dynein/dynactin deficiency probably causes morphogenetic effects by (1) diminishing the traffic of secretory vesicles from their synthesis site (endoplasmic reticulum) either to intermediate secretory compartments or to the hyphal apex; (2) affecting the organization and movement of the growing microtubules; and/or (3) impeding the proper recycling of material needed for normal apical growth to proceed.

\section{ACKNOWLEDGEMENTS}

We would like to thank Dr Stuart Brody from the University of California, San Diego, and Dr Leah Haimo from the University of California, Riverside, for helpful suggestions during the course of this work. Ms Riquelme was supported by a Research Assistantship from the University of California, Riverside.

\section{REFERENCES}

Bartnicki, D. D., Gierz, G. \& Bartnicki-García, S. (1994). "Fungus Simulator": a Windows application to model fungal morphogenesis. In Abstracts of the 5th International Mycological Congress, Vancouver, Canada, p. 12.

Bartnicki-García, S. (1968). Cell wall chemistry, morphogenesis, and taxonomy of fungi. Annu Rev Microbiol 22, 87-107.

Bartnicki-García, S. (1973). Fundamental aspects of hyphal morphogenesis. In Microbial Differentiation, pp. 245-267. Edited by J. M. Ashworth \& J. E. Smith. Cambridge: Cambridge University Press.

Bartnicki-García, S. \& Lippman, E. (1969). Fungal morphogenesis. Cell wall construction in Mucor rouxii. Science 165, 302-304.

Bartnicki-García, S., Hergert, F. \& Gierz, G. (1989). Computer simulation of fungal morphogenesis and the mathematical basis for (hyphal tip) growth. Protoplasma 153, 46-57.

Bartnicki-García, S., Bartnicki, D. D., Gierz, G., López-Franco, R. \& Bracker, C. E. (1995). Evidence that Spitzenkörper behavior determines the shape of a fungal hypha: a test of the hyphoid model. Exp Mycol 19, 153-159.

Bourett, T. M. \& Howard, R. J. (1991). Ultrastructural immunolocalization of actin in a fungus. Protoplasma 163, 199-202.

Bracker, C. E. (1995). The video-enhanced light microscope: a renaissance tool for quantitative live-cell microscopy. Zool Stud 34, 154-156.

Bracker, C. E., Murphy, D. J. \& López-Franco, R. (1997). Laser microbeam manipulation of cell morphogenesis in growing fungal hyphae. In Functional Imaging and Optical Manipulation of Living Cells, pp. 67-80. Edited by D. L. Farkas \& B. J. Tromberg. Bellingham, WA: SPIE (International Society for Optical Engineering) (Proceedings of SPIE vol. 2893).

Bruno, K. S., Tinsley, J. H., Minke, P. F. \& Plamann, M. (1996). Genetic interactions among cytoplasmic dynein, dynactin, and nuclear distribution mutants of Neurospora crassa. Proc Natl Acad Sci USA 93, 4775-4780.

Garnjobst, L. \& Tatum, E. L. (1967). A survey of new morphological mutants in Neurospora crassa. Genetics 57, 579-604.

Gill, S. R., Schroer, T. A., Szilak, I., Steuer, E. R., Sheetz, M. P. \& Cleveland, D. W. (1991). Dynactin, a conserved, ubiquitously expressed component of an activator of vesicle motility mediated by cytoplasmic dynein. J Cell Biol 115, 1639-1650.

Girbardt, M. (1957). Der Spitzenkörper von Polystictus versicolor (L.). Planta 50, 47-59.

Girbardt, M. (1969). Die Ultrastruktur der Apikalregion von Pilzhyphen. Protoplasma 67, 413-441.

Gooday, G. W. (1983). The hyphal tip. In Fungal Differentiation, pp. 315-356. Edited by J. E. Smith. New York: Marcel Dekker.

Gow, N. A. R. (1989). Control of the extension of the hyphal apex. Curr Top Med Mycol 3, 109-152.

Grove, S. N. \& Bracker, C. E. (1970). Protoplasmic organization of hyphal tips among fungi. J Bacteriol 104, 989-1009. 
Haimo, L. T. \& Thaler, C. D. (1994). Regulation of organelle transport: lessons from color change in fish. BioEssays 16, 727-733.

Harold, F. M. (1990). To shape a cell: an inquiry into the causes of morphogenesis of microorganisms. Microbiol Rev 54, 381-431.

Hasek, J. \& Bartnicki-García, S. (1994). The arrangement of F-actin and microtubules during germination of Mucor rouxii sporangiospores. Arch Microbiol 161, 363-369.

Heath, I. B. (1994). The cytoskeleton in hyphal growth, organelle movements, and mitosis. In The Mycota I: Growth, Differentiation and Sexuality, pp. 43-65. Edited by J. G. H. Wessels \& F. Meinhardt. Berlin \& Heidelberg: Springer.

Hirokawa, N. (1982). Cross-linker system between neurofilaments, microtubules and membranous organelles in frog axons revealed by the quick-freeze, deep-etching method. J Cell Biol 94, 129-142.

Hirokawa, N. (1998). Kinesin and dynein superfamily proteins and the mechanism of organelle transport. Science 279, 519-526.

Hoch, H. C. \& Staples, R. C. (1985). The microtubule cytoskeleton in hyphae of Uromyces phaseoli germlings: its relationship to the region of nucleation and to the F-actin cytoskeleton. Protoplasma 124, 112-122.

Hoffmann, J. \& Mendgen, K. (1998). Endocytosis and membrane turnover in the germ tube of Uromyces fabae. Fungal Genet Biol 24, 77-85.

Howard, R. J. (1981). Ultrastructural analysis of hyphal tip cell growth in fungi: Spitzenkörper, cytoskeleton and endomembranes after freeze-substitution. J Cell Sci 48, 89-103.

Howard, R. \& Aist, J. R. (1977). Effects of MBC on hyphal tip organization, growth, and mitosis of Fusarium acuminatum, and their antagonism by $\mathrm{D}_{2} \mathrm{O}$. Protoplasma 92, 195-210.

Howard, R. J. \& Aist, J. R. (1980). Cytoplasmic microtubules and fungal morphogenesis: ultrastuctural effects of methyl benzimidazole-2-ylcarbamate determined by freeze-substitution of hyphal tip cells. J Cell Biol 87, 55-64.

Inoue, S., Turgeon, B. G., Yoder, O. C. \& Aist, J. R. (1998). Role of fungal dynein in hyphal growth, microtubule organization, spindle pole body motility and nuclear migration. J Cell Sci 111, 1555-1566.

Lehmler, C., Steinberg, G., Snetselaar, K. M., Schliwa, M., Kahmann, R. \& Bölker, M. (1997). Identification of a motor protein required for filamentous growth in Ustilago maydis. EMBO J 16, 3464-3473.

López-Franco, R. (1992). Organization and dynamics of the Spitzenkörper in growing hyphal tips. PhD dissertation, Purdue University, West Lafayette, IN.

López-Franco, R. \& Bracker, C. E. (1996). Diversity and dynamics of the Spitzenkörper in growing hyphal tips of higher fungi. Protoplasma 195, 90-111.

McClure, W. K., Park, D. \& Robinson, P. M. (1968). Apical organization in the somatic hyphae of fungi. J Gen Microbiol 50, 177-182.

McKerracher, L. J. \& Heath, I. B. (1987). Cytoplasmic migration and intracellular organelle movements during tip growth of fungal hyphae. Exp Mycol 11, 79-100.

Marsden, J. E., Tromba, A. J. \& Weinstein, A. (1993). Basic Multivariable Calculus. New York: Springer.

Paschal, B. M., Shpetner, H. S. \& Vallee, R. B. (1987). MAP 1C is a microtubule-activated ATPase which translocates microtubules in vitro and has dynein-like properties. J Cell Biol 105, 1273-1282.
Plamann, M., Minke, P. F., Tinsley, J. H. \& Bruno, K. S. (1994). Cytoplasmic dynein and actin-related protein Arp1 are required for normal nuclear distribution in filamentous fungi. J Cell Biol 127, 139-149.

Reiss, H. D. \& Herth, W. (1979). Calcium gradients in tip growing plant cells visualized by chlorotetracycline fluorescence. Planta 146, 615-621.

Reynaga-Peña, C. G. \& Bartnicki-García, S. (1997). Apical branching in a temperature sensitive mutant of Aspergillus niger. Fungal Genet Biol 22, 153-167.

Riquelme, M., Reynaga-Peña, C. G., Gierz, G. \& Bartnicki-García, S. (1998). What determines growth direction in fungal hyphae? Fungal Genet Biol 24, 101-109.

Roberson, R. W. \& Vargas, M. M. (1994). The tubulin cytoskeleton and its sites of nucleation in hyphal tips of Allomyces macrogynus. Protoplasma 182, 19-31.

Schroer, T. A. \& Sheetz, M. P. (1991). Two activators of microtubule-based vesicle transport. J Cell Biol 115, 1309-1318.

Seiler, S., Nargang, F. E., Steinberg, G. \& Schliwa, M. (1997). Kinesin is essential for cell morphogenesis and polarized secretion in Neurospora crassa. EMBO J 16, 3025-3034.

Seiler, S., Plamann, M. \& Schliwa, M. (1999). Kinesin and dynein mutants provide novel insights into the roles of vesicle traffic during cell morphogenesis in Neurospora. Curr Biol 9, 779-785.

Sievers, A. (1963). Beteiligung des Golgi-Apparates bei der Bildung der Zellwand von Wurzelhaaren. Protoplasma 56, 188-192.

Steinberg, G. \& Schliwa, M. (1996). Characterization of the biophysical and motility properties of kinesin from the fungus Neurospora crassa. J Biol Chem 271, 7516-7521.

Tinsley, J. H., Minke, P. F., Bruno, K. S. \& Plamann, M. (1996). $\mathrm{p} 150^{\mathrm{Glu}}$, the largest subunit of the dynactin complex, is nonessential in Neurospora but required for nuclear distribution. Mol Biol Cell 7, 731-742.

Trinci, A. P. J. (1978). Wall and hyphal growth. Sci Prog 65, 75-79. Turian, G. (1978). The "Spitzenkörper", centre of the reducing power in the growing hyphal apices of two septomycetous fungi. Experientia 34, 1277-1279.

Vale, R. D., Reese, T. S. \& Sheetz, M. P. (1985). Identification of a novel force-generating protein, kinesin, involved in microtubulebased motility. Cell 42, 39-50.

Vierula, P. J. (1996). The genetics of morphogenesis in Neurospora crassa. In Patterns in Fungal Development, pp. 87-104. Edited by S.-W. Chiu \& D. Moore. Cambridge: Cambridge University Press.

Vogel, H. J. (1956). A convenient growth medium for Neurospora (Medium N). Microb Genet Bull 13, 42-43.

Wu, Q., Sandrock, T. M., Turgeon, B. G., Yoder, O. C., Wirsel, S. G. \& Aist, J. R. (1998). A fungal kinesin required for organelle motility, hyphal growth, and morphogenesis. Mol Biol Cell 9, 89-101.

Xiang, X., Beckwith, S. M. \& Morris, N. R. (1994). Cytoplasmic dynein is involved in nuclear migration in Aspergillus nidulans. Proc Natl Acad Science USA 91, 2100-2104.

Xiang, X., Roghi, C. \& Morris, N. R. (1995). Characterization and localization of the cytoplasmic dynein heavy chain in Aspergillus nidulans. Proc Natl Acad Sci USA 92, 9890-9894.

Received 5 January 2000; revised 29 March 2000; accepted 25 April 2000. 